\title{
INFECTION IN THE NEWBORN BABY
}

\author{
By Alan MoncriefF, M.D., F.R.C.P. \\ Nuffield Professor of Child Health, University of London; Physician, Tine Hospital for Sick Children, \\ Great Ormond Street, London, W.C. I
}

Infection in the newborn baby has not had the recognition that it deserves. Mortality figures give it a relatively low place in the list of causes of death in the neonatal period. Nevertheless limited series of carefully worked out statistics based upon expert post-mortem examinations indicate that infection has been responsible for the death of well over a third and possibly for as many as a half of all babies dying in the early weeks of life. This is particularly unfortunate nowadays when chemotherapy offers a valuable weapon against neonatal sepsis-provided, of course, that it is accurately diagnosed. There seem to be two reasons why such diagnosis is not more often made. First, there is a somewhat hazy idea that newborn babies are immune-that they 'don't catch things,' and secondly the clinical picture of infection in the small infant differs considerably from the more familiar condition in older children or adults.

The whole question of immunity in the newborn is fascinating, connected on the one hand with the route of transmission from the mother, placental or by colostrum and milk, and on the other with the level of plasma proteins in the infant. Many problems remain to be settled and deductions from animal experiments may be invalid if the placental structure shows much species difference from that of the human. Leaving this all on one side, however, it can be confidently asserted that a baby can only possess at the best such immunity as the mother herself enjoys. Whereas this may be high as regards the infectious fevers and explains why such disorders as measles, pertussis and diphtheria for example are rare in the newborn, it is almost certain to be low or non-existent to such infections as the 'common cold' and the pyogenic organisms. It is with such infections that this article is mostly concerned.

The introduction of infection may be before, during or after birth. Infection in utero is rare and the only common disorder that is likely to be acquired in this way is syphilis. There are occasional instances in the literature of transplacental transmission of tuberculosis, and circumstantial evidence suggest that $B$. coli can sometimes pass this way. But such congenital infections are not of great importance with the exception of syphilis, which could and should be easily prevented by a routine serological examination as part of the antenatal programme, followed by treatment in appropriate cases.

During birth the most important infection is by the gonococcus, producing ophthalmia. The use of silver nitrate for babies' eyes has in the past, when efficiently used, proved an adequate preventive measure. It had, however, certain disadvantages and in view of the extremely effective and rapid cure of gonococcal ophthalmia by penicillin systemically administered, some centres are now abandoning the use of prophylactic drops at birth. During the birth process the doctor or nurse is in fairly intimate contact with the baby and there is evidence that some infections, such as the common cold or sore throats or staphylococcal skin infection, may be transmitted at this time unless a careful aseptic routine for delivery of the baby is adopted. Such infections merge into the next group.

Infection after birth must be viewed in light of modern facts and not on the basis of an almost preListerian outlook. This remark is necessary because over and over again in discussions on this subject with doctors and midwives, it transpires that infection of the newborn baby means for most of them umbilical sepsis. In civilized countries this is extremely rare. Whereas the commonest of all infections, the 'common cold' and upper respiratory tract infections in general, are seldom mentioned. Yet it is the latter group and not the former, which is the major problem in neonatal work. The portals of entry of infection may be listed more or less in order of frequency. First comes the respiratory tract (mouth, nose) spreading to the lower respiratory tract on the one hand, and on the other to the alimentary tract, producing here the alarming epidemic neonatal diarrhoea. Next must be put the skin, but probably only when, because of abrasions for example, it ceases to be an intact barrier. Lastly the umbilicus, the eyes and rarely the genitalia.

The evolution of infection at the portal of entry may be very easily missed and this is especially the case where the nose is involved. The bab: 
lying flat, has a type of ' cold' which is most unlike that of adults. The posterior nares and nasopharynx are affected more than the anterior nares. A minimum of nasal discharge-anteriorly-is noted. There is little or no sneezing. There is obviously no tell-tale pile of pocket handkerchiefs! Some degree of nasal obstruction may occur, leading to attacks of cyanosis and respiratory distress, sometimes, but by no means always, associated with ' snuffles' or noisy nasal breathing. In other situations as for example on the skin or the umbilical stump, the primary changes may be obvious; but even for these areas, as for the common cold, it may be that the secondary stage of generalization, with or without local spread, is the first abnormality to be noted.

Nor are the manifestations of generalized sepsis easy to recognize on the basis of what is seen in adult medicine. Fever in the usual sense may not be observed. This is sometimes because the newborn baby fails to respond to toxins by a rise in temperature and may even react by developing severe subnormal levels before death. But there is also the point that fever in the sense of a rise in temperature may be present but be unrecognized as such. This paradox is explained by the fact that the " normal ' level of temperature in the newborn baby and especially in the premature infants is often two or more degrees below the usually accepted adult level of $99^{\circ} \mathrm{F}$. or so per rectum. Suppose the baby to have been running at a rectal temperature of $96^{\circ}$ or $97^{\circ} \mathrm{F}$. A rise of two degrees will bring it only just up to the 'normal' of adult reckoning. Such a rise in an adult would mean a recording of $\mathrm{rOI}^{\circ}$ or sofever would undoubtedly be recognized. This difficulty over rises in temperature is another reason why sepsis in the newborn is so often missed.

The next manifestations of septicaemia-for this is what the condition has now becomeare a reluctance to feed (possibly partly because of a blocked nasal airway), vomiting and sometimes diarrhoea. The weight curve stops rising and often falls steeply. The pulse becomes rapid, respirations are increased in rate and often become irregular and the baby develops a grey, dehydrated appearance which is ominously characteristic. Oedema is another curious phenomenon which sometimes appears at this stage. If the infant survives and treatment has not-or even if it has -been instituted, a third stage of pyaemic manifestations may now set in. This leads to an even more complicated and bizarre picture. Multiple skin abscesses may appear, pemphigus-like blisters develop, jaundice, a septic bronchopneumonia, pyelo-nephritis, epiphysitis and arthritis, mening- itis are some of the chief complications seen at this stage. Otitis media is a continual menace, probably because of the frequent naso-pharyngeal origin of the sepsis, the vomiting and the flat position in which the baby lies. Rarities which have achieved eponymous notoriety are Winckel's disease with haemoglobinuria and Bühl's disease with fatty degeneration of the liver.

Treatment must start with prevention; a $a^{\perp}$ ter such a depressing summary as that just given it is clear that the best programme is one which keeps invading micro-organisms away from a host with so little resistance and such an unfortunate tendency to generalize infection when it occurs. This is not the place to lay down details of obstetric methods, but those who handle a newborn baby have a responsibility to see that what may be minor or trivial infections somewhere in their person, do not get transferred to the infant. For example, masking should certainly be employed if there is the slightest suspicion of acute or chronic infections of the upper respiratory tract. This applies to the mother as well as to doctor and midwife. I have seen the four-pound baby of an ear, nose and throat surgeon very nearly die of bronchopneumonia where the source of infection was the mother's nasal antrum, and a small outbreak of staphylococcal pneumonia in the new? born, before the days of chemotherapy and there fore highly fatal, was traced to staphylococci in? the noses of nurses in the wards in question. Virus infections of the nose of those handling the baby are less easy to trace, epidemiologically speaking, than the pyogenic organisms but are probably more prevalent and important. Similarly great care must be taken over small septic spots about the hands. One outbreak of pemphigus was traced after investigation to a laundry worker with a healing whitlow who was folding the babies' clothes after washing.

If infection occurs, then treatment can be considered under three main headings. First, breast feeding or feeding with pooled breast milk must be maintained as far as possible. It is important not to add digestive disturbances to the baby's troubles. Next, if possible, the infecting organism should be identified, by nasal and post-nasal swabbing, by blood culture or by culture of obvious skin or umbilical lesions. Pending the results of this, treatment by penicillin systemically or by mouth-since young infants apparently absorb easily from the alimentary tract, should be instituted. The accepted dosage for intramuscular injection is 2,000 units per pound body weight per day divided into four-hourly doses and by mouth at least twice the dose by the intramuscular route. Some authorities add a sulphonamide preparation 
at the outset, such as sulphamezathine by mouth in doses of $\mathrm{I}$ to 2 grains per pound body weight per day in four-hourly doses. But if there is likely to be only a short delay in bacteriological diagnosis it may be better to wait for this and adjust the programme accordingly. If the very rare $B$. coli infection is found, i.e., a peritonitis or meningitis, streptomycin should be considered. The third step is the general treatment of the baby. Expert nursing is essential, and the prevention of superadded infection. The baby should be isolated and if the illness is at all severe then the expert services of a children's hospital or department should be sought. A watch must be kept on the state of hydration, especially if diarrhoea and vomiting complicate the picture, and steps taken, as by intravenous fluids, to remedy any serious de- hydration found. Similarly a watch must be kept on the haemoglobin level. Neonatal sepsis often leads to an increased haemolysis and in the presence of anaemia the baby has less chance of dealing with the infection. Iron may be given by mouth in the mild cases or a blood transfusion used if the haemoglobin drops below, say, 50 per cent. Haldane. Local treatment of abscesses and skin sepsis should follow accepted lines.

With this programme there is every prospect of success, save in the severer cases and in the smaller and possibly premature infants. Once recovery has been secured there should be no residual damage. Babies who are saved from death from neonatal sepsis have every prospect of growing up into normal and useful citizens.

\title{
THE ANAEMIAS OF INFANCY
}

\author{
By JaMes H. Hutchison, O.B.E., M.D., F.R.C.P., F.R.F.P.S.G. \\ Visiting Physician, Royal Hospital for Sick Children, Glasgow
}

Anaemia is one of the commonest causes of morbidity during the period of infancy. Baty (1947) analysed I,500 consecutive admissions to the Boston Floating Hospital ; 514 patients (34 per cent.) showed a definite anaemia, which in 92 per cent. was the result of infection, dietary deficiency, prematurity, or a combination of these factors. This analysis confirms the fact, already well-known to paediatricians, that the anaemias associated with iron deficiency are by. far the commonest of the blood diseases of infancy. Irondeficiency anaemia will, therefore, receive detailed consideration in this review. It is proposed also to discuss those anaemias of infancy in which recent advances have been made, leaving out of consideration anaemias rarely encountered in this country, e.g. Cooley's anaemia, sickle-cell anaemia, etc., and blood diseases in which anaemia is not the basic feature.

Any discussion on the subject of anaemia in infancy must be prefaced by some consideration of the normal range of values of the various blood components during this period. Failure to appreciate that these values differ from those accepted as physiological in later years has led frequently to errors in diagnosis.

\section{Haemoglobin Levels in the First Year of Life}

The extensive researches of Mackay (I93 I, r933(b)) produced the first authoritative and generally accepted British figures for the $\mathrm{Hb}$ content of the blood of infants during the first year of life (Fig. I). The estimations were made with a standardized Haldane haemoglobinometer in which 100 per cent. was assumed to be equivalent to $13.8 \mathrm{gm}$. of $\mathrm{Hb}$ per $100 \mathrm{cc}$. of blood. King and his co-workers (1947) by accepting iron determination as the most reliable method for estimating $\mathrm{Hb}$ have shown, however, that the modern B.S.I. Haldane-Gowers $\mathrm{Hb}$ Standard-British Standard I079 (1942)-is equivalent to $\mathrm{r}_{4} .8 \mathrm{gm}$. of $\mathrm{Hb}$, and not $13.8 \mathrm{gm}$. as previously assumed. It is, therefore, permissible to convert Mackay's (I933(b)) figures into terms of grammes of $\mathrm{Hb}$ per cent. on the assumption that a reading of roo per cent. on her haemoglobinometer was also equivalent to I4.8 gm. per cent.

It will be seen that the normal $\mathrm{Hb}$ level at birth is 143 per cent. (20.2 $\mathrm{gm}$. Hb per cent.). That this falls to 106 per cent. (15.7 gm.) in the first two weeks of life, and reaches 74 per cent. (II gm.) at three months. Thereafter it rises gradually to 